\title{
On- and Off-Responses in the Inferior Colliculus of the Greater Horseshoe Bat to Pure Tones*
}

\author{
Gerhard Neuwetler, Gerd Schuller, and Hans-Ulutch Schnitzler \\ Zoophysiologisches Institut der Universität Tübingen
}

Received May 14, 1971

\begin{abstract}
Summary. 1. The auditory threshold curve of averaged evoked potentials of the colliculus inferior in Rhinolophus ferrum equinum to pure tone stimulation from 1 to $100 \mathrm{kHz}$ is presented (Fig. 1). For pure tone frequencies lower than $14 \mathrm{kHz}$ thresholds steeply rise from 20 to $110 \mathrm{db}$. The steepness of the acoustical filter for $83.3 \mathrm{kHz}$ signals and the frequency of the echoes heard by Greater Horseshoe Bats is accentuated by averaging methods. Because of averaging, evoked potentials thresholds are lowered by about $20 \mathrm{db}$ compared to those obtained by non-averaging methods.

2. Prominent off-responses in evoked potentials appear for three stimulus frequency ranges: $3-10 \mathrm{kHz}$ with a peak at $5 \mathrm{kHz}, 20-40 \mathrm{kHz}$ with a peak at $20 \mathrm{kHz}$ and $80.0-82.6 \mathrm{kHz}$ with a peak at $81.5 \mathrm{kHz}$ (Fig. 2).

3 . It is unlikely that beat note frequencies play any role for echolocation.

4. The relevance of these results to echolocation in Horseshoe Bats is discussed, especially as to Doppler shift information contained in the constant frequency part of echoes.
\end{abstract}

\section{Introduction}

Horseshoe Bats emit long ultrasonic orientation sounds of constant frequency with a terminal frequency-drop of about $14-16 \mathrm{kHz}$. Flying bats lower the frequency of the constant frequency part (CF) by an amount which compensates for Doppler shifts caused by the flight velocity. The frequency is therefore kept constant at about $83.3 \mathrm{kHz}$ which is the frequency of resting bats (Schnitzler, 1968). At this frequency the auditory threshold of on-responses of collicular evoked potentials has a minimum and rises steeply at the frequencies below and above (Neuweiler, 1970). This filter for $83.3 \mathrm{kHz}$ stimuli may be realized by peripheral, basilar or nervous processes or combinations of them. In this paper we report collicular off-responses to pure tone stimulation occurring in restricted frequency ranges which may indicate that, at least partially, central nervous processes are involved in the filter for $83.3 \mathrm{kHz}$.

* This study was supported by Stiftung Volkswagenwerk, grant No. 110771 and DFG, grant No. Va 22/4. 
The Doppler shift compensation in connection with the acoustical filter diminishes the problem of masking of overlapping outgoing pulses and returning echoes. According to a theory of J. D. Pye $(1960,1961)$ and L. Kay $(1961,1962)$ the overlapping would account for a different way of evaluating frequency shifts caused by Doppler effects. In their theory, the simultaneous hearing of emitted sound and returning echo would produce a beat note, the frequency of which is proportional to the flight velocity of the bat relative to the target. The frequency of the beat note may range from a few $\mathrm{Hz}$ to $3 \mathrm{kHz}$. Pye assumes that the source of nonlinearity for creation of beat notes are the middle ear muscles. In that case the auditory system of Horseshoe Bats should be sensitive to low frequency stimuli. We therefore extended the stimulus frequency range for threshold measurements in the colliculus inferior into the audible range down to $1 \mathrm{kHz}$.

\section{Methods}

Auditory evoked potentials from 7 Nembutal-anesthetized Rhinolophus ferrum equinum were recorded in the colliculus inferior using Ag-AgCl-electrodes. Thresholds of on-responses were determined for pure tone stimulation from 1 to $100 \mathrm{kHz}$. The experimental procedure is basically the same as described in Neuweiler (1970).

For stimulation in the range from $1-20 \mathrm{kHz}$ we used a Valvo AD $1260 \mathrm{M}-5$ loudspeaker and for ultrasonic frequencies a condensator loudspeaker of our own design.

For each set of stimulus parameters 100 evoked potentials were averaged by a Didac-800 computer (Intertechnique). Stimulus repetition rate was $8-9 / \mathrm{sec}$ while stimulus duration lasted $30 \mathrm{msec}$ with a rise- and fall-time of 2 msec. Stimulus intensity is expressed in $\mathrm{db}$ re $0.0002 \mathrm{dyn} / \mathrm{cm}^{2}$. The analysis time for 800 channels was $96 \mathrm{msec}$. Thresholds were measured by lowering the sound intensity in $5 \mathrm{db}$ steps and determined by the intensity at which a response was no longer visible. The amplitude of the averaged evoked responses was left uncalibrated.

\section{Results}

\section{Thresholds of On-Responses}

Compared to the conventional method used in the previous study (Neuweiler, 1970) the averaging method results in an overall decrease of detectable collicular thresholds for pure tone stimulation of about $20 \mathrm{db}$. Fig. 1 shows a representative example of the recorded threshold curves. The lowest threshold in the ultrasonic frequency range extends to $-5 \mathrm{db}$ as compared to $30-25 \mathrm{db}$ in nonaveraged studies.

Invariably in all threshold curves a narrowly tuned filter for $83.3 \mathrm{kHz}$ signals appears with a steeply rising slope for lower frequencies down to $81.5 \mathrm{kHz}$ (see inset in Fig. 1). The frequencies of highest and lowest thresholds are consistently $81.5-81.8 \mathrm{kHz}$ and $83.3-83.5 \mathrm{kHz}$ respectively. By the averaging method the threshold difference between 81.5 and 


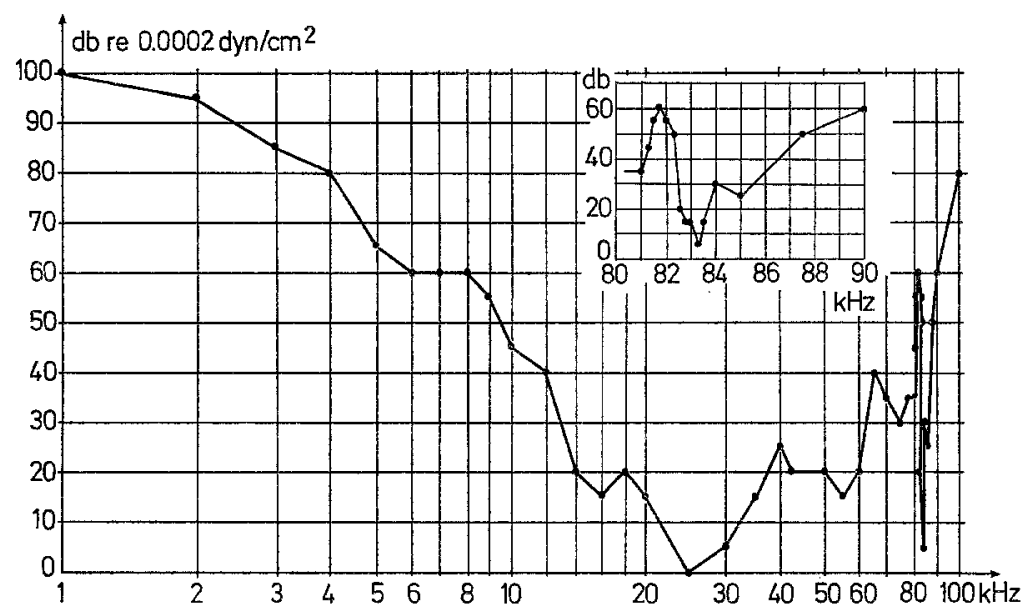

Fig. 1. Threshold curve of averaged evolked potentials of the inferior colliculus in Rhinolophus ferrum equinum (RF 5/14/7). Abscissa: stimulus frequency; ordinate: threshold in $\mathrm{db}$ re $0.0002 \mathrm{dyn} / \mathrm{cm}^{2}$. Stimulus: pure tone, 30 msec duration, $2 \mathrm{msec}$ rise/fall time, $8-9 /$ sec repetition rate. Inset: Enlarged section of the threshold curve for stimulus-frequencies from 80.0 to $90.0 \mathrm{kHz}$

$83.3 \mathrm{kHz}$ signals is accentuated compared to those of unaveraged responses. The threshold for $81.5 \mathrm{kHz}$ tones always is $50-60 \mathrm{db}$ and for $83.3 \mathrm{kHz}$ tones $10-5 \mathrm{db}$. This amounts to an average threshold slope of $40-55 \mathrm{db} / 1.8 \mathrm{kHz}$ compared to a $33 \mathrm{db} / 1.8 \mathrm{kHz}$ slope in former experiments (Neuweiler, 1970). At frequencies higher than $83.3-83.5 \mathrm{kHz}$ thresholds again rapidly increase but less steeply than those for lower frequencies.

Low thresholds of frequencies other than $83.3 \mathrm{kHz}$ are more variable. An absolute minimum of threshold for stimuli between 20 and $30 \mathrm{kHz}$ exists in most threshold curves (see Fig. 1). In two cases however this minimum occurred for frequencies between 60 and $70 \mathrm{kHz}$. Invariably there appears a small maximum of threshold for $40 \mathrm{kHz}$ tones.

As stimulus frequency is lowered from 20 to $10 \mathrm{kHz}$ thresholds of collicular evoked potentials increase by $20-30 \mathrm{db}$ (see Fig. 1). The low thresholds for $10 \mathrm{kHz}$ tones recorded in the previous paper (Neuweiler, 1970) most probably result from stimulus distortions caused by the loudspeaker for frequencies below $20 \mathrm{kHz}$.

From 8 to $5 \mathrm{kHz}$ the threshold curve remains flat at a high level of $60-70 \mathrm{db}$. For still lower frequencies down to $1 \mathrm{kHz}$ the thresholds rise again by $25-40 \mathrm{db}$ and reach a value of $110 \mathrm{db}$ which is the highest intensity available in our loudspeaker. In two bats the maximal intensity was not sufficient to elicit any collicular response for 1 and $2 \mathrm{kHz}$ tones. 


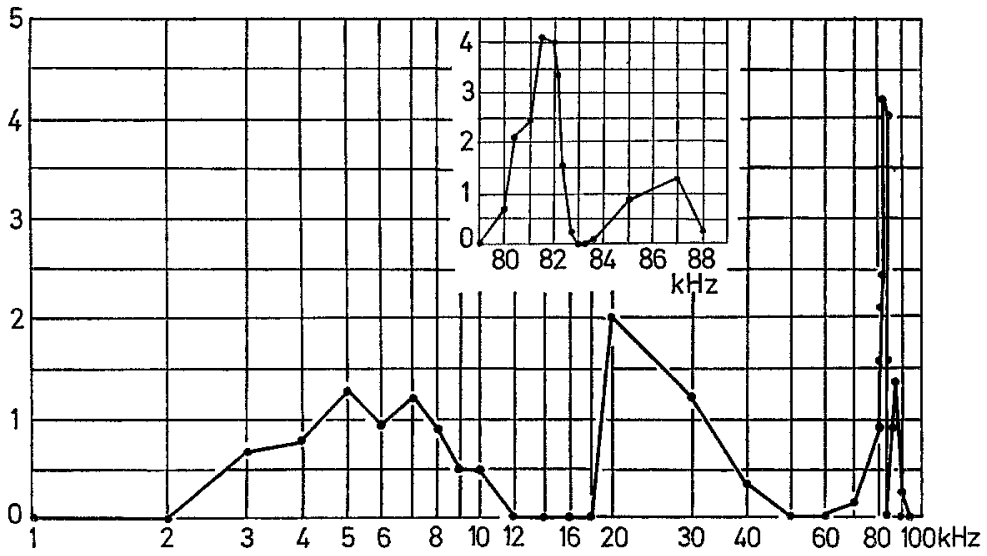

Fig. 2. Amplitude of off-responses in averaged collicular evoked responses of Rhinolophus ferrum equinum. Each point is the average of 3 experiments. Abscissa: stimulus frequency; ordinate: amplitude of the off-response in arbitrary units. Stimulus: pure tone, $30 \mathrm{msec}$ duration, $2 \mathrm{msec}$ rise/fall time, intensity $70 \mathrm{db}$ re $0.0002 \mathrm{dyn} / \mathrm{cm}^{2}, 8-9$ sec repetition rate. Inset: Enlarged section of the amplitude curve for stimulus frequencies from 80.0 to $90.0 \mathrm{kHz}$

If one interprets thresholds of collicular responses as correlates of hearing thresholds, Horseshoe Bats may detect auditory signals in the frequency range of $12-5 \mathrm{kHz}$ only at high intensities and frequencies below $4 \mathrm{kHz}$ will not be heard under natural conditions.

\section{Off-Responses}

Off-responses to pure tone stimulation are an uncommon feature in the auditory system of mammals. In bats they only occur in species which emit a CF-part in their echolocation pulses (Grinnell, 1970).

Horseshoe Bats are no exception to Grinnell's statement since at certain frequency bands prominent off-responses appear in the evoked potentials of the colliculus inferior. Fig. 2 shows the mean amplitude of off-responses plotted as a function of stimulus frequency from 3 bats. Stimulus intensity was $70 \mathrm{db}$. There are three distinet maxima of offresponses, the first from $3-10 \mathrm{kHz}$, with a peak at $5 \mathrm{kHz}$, the second from 20 to $40 \mathrm{kHz}$ with peak amplitude at $20 \mathrm{kHz}$ and the third from 80.0 to $82.6 \mathrm{kHz}$ with peak amplitude at $81.5 \mathrm{kHz}$. Viewed on a logarithmic scale the frequency range for off-responses gets narrower with increasing stimulus frequency and the amplitude peak quickly increases from low to high frequency range.

If one takes the lowest frequency producing a maximal off-response as the fundamental one, it seems that the maxima of off-responses occur at harmonics of the basic frequency. 
The most interesting feature of the off-effects again appears in the frequency range of the echolocation pulses (see inset in Fig. 2). The maximal off-amplitude occurs exactly at $81.5 \mathrm{kHz}$, the frequency of highest threshold for on-responses. With increasing frequency the offresponses rapidly decline and reach a minimum at the echofrequency of $83.0-83.3 \mathrm{kHz}$ where threshold of on-responses is minimal. At this frequency for very high sound pressure levels only a small off-effect may be obtained. For stimulus frequencies from $84-88 \mathrm{kHz}$ the off-responses increase again but never reach the amplitude of those for $81.5 \mathrm{kHz}$ stimuli.

Off-responses may be elicited by stimulus frequencies other than those in the three frequency ranges mentioned, but then extremely high intensities have to be applied. For 50 and $60 \mathrm{kHz}$ stimuli we never obtained off-responses even at $110 \mathrm{db}$, the highest intensity available.

\section{Discussion}

This study confirms and validates the existence of an acoustical filter at the frequency of the CF-part of the echo heard by a Greater Horseshoe Bat. The significance of this filter for echolocation in corroboration with Doppler shift compensation has been discussed elsewhere (Neuweiler, 1970). The main points are shortly summarized here:

Flying bats lower the frequency of the CF-part of the emitted sound in order to compensate for Doppler shifts. This frequency therefore varies between 81.0 and $83.3 \mathrm{kHz}$ dependent on flight velocity whereas the echofrequency is kept constant at $83.3 \mathrm{kHz}$ (Schnitzler, 1968). By using a narrowly tuned filter for this echo-frequency of $83.3 \mathrm{kHz}$, collicular thresholds for the CF-part of the echo are very low whereas thresholds for the frequencies of the emitted sounds are high (see inset in Fig. 1). Thus masking of the echo by ongoing sound emission may be avoided.

Doppler shifts are most prominent for echoes returning from flight direction $\left(0^{\circ}\right)$. They decrease by the cosinus for other angles of incidence and are zero for echoes returning perpendicular to flight direction. Since the emitted frequency is regulated for maximal Doppler shifts in the echoes, i.e. for echoes from $0^{\circ}$-direction, the frequencies of the echoes from greater angles are lower than $83.3 \mathrm{kHz}$ and fall in an area of high hearing thresholds. Therefore the acoustical filter results in a preferred processing of echoes returning from straight ahead.

The most surprising feature of the Horseshoe Bat's threshold curve is not the low threshold for the echo-frequency at $83.3 \mathrm{kHz}$ but the very high one for the frequency range of the emitted sounds at $81.5 \mathrm{kHz}$. This "sharply tuned" frequency range of high thresholds separates 
hearing of the CF-part of the echoes from hearing of any lower frequency. How is this high threshold established? Since it is so narrowly tuned to $81.5 \mathrm{kHz}(Q$-value 81.5$)$, it is tempting to postulate neural inhibition. If one considers off-responses as rebound from inhibition this postulate is strengthened by the very fact that the most prominent off-responses exactly appear for frequencies around $81.5 \mathrm{kHz}$ whereas for $83.3 \mathrm{kHz}$ off-effects reach a minimum or are absent. A drawback, however, is that off-responses also occur at 20 and $5 \mathrm{kHz}$ where neural inhibition seems to be unnecessary.

Grinnell (1970) described prominent off-responses in Chilonycteris rubiginosa, a bat emitting a long CF-part of $64 \mathrm{kHz}$. Just below the frequency of the bat's greatest sensitivity a sharply tuned off-maximum at $60-62 \mathrm{kHz}$ exists which again coincides with a maximum of threshold for on-responses. As Chilonycteris rubiginosa compensates for Dopplershifts (Schnitzler, 1970), it appears that this bat also has an acoustical filter tuned to its echo frequency of $64 \mathrm{kHz}$.

Grinnell interprets the off-responses of the CF-part as time markers which enable a bat emitting long pure tone pulses to measure the time difference between sound emission and echo for detection of target range. This interpretation does certainly not apply to Horseshoe Bats since in these animals there exists an absolute minimum of off-responses for the very echo frequency of $83.3 \mathrm{kHz}$ (see inset in Fig. 2). On the basis of the available experimental data we consider off-responses in the colliculus inferior to pure tone stimulation as a manifestation of neural inhibition.

Information contained in Doppler shifts may be carried by beat notes produced by simultaneous hearing of emitted frequency and the Doppler shifted echo (Pye, 1960, 1961; Kay, 1961, 1962). For Horseshoe Bats this theory seemed plausible since emitted echolocation sound and returning echo overlap for target ranges within about $10 \mathrm{~m}$. Pye considers this to be caused by nonlinearity in the middle ear (muscle activity) which produces a difference tone from the two primary frequencies. In this case Horseshoe Bats should have low hearing thresholds for frequencies of a few $\mathrm{Hz}$ to $3 \mathrm{kHz}$. As Fig. 1 shows thresholds in fact are very high at low frequencies. Only when the site of nonlinearity is shifted to the basilar membrane (Tonndorf, 1959) hearing of beat note frequencies seems possible. If the insensitivity to low frequencies, as shown in the threshold curve, is caused by a high pass filter in the sound transmitting system of the middle ear, this barrier for low frequencies may be overcome by beat notes produced at the basilar membrane. According to Tonndorf (1959) similar high intensities of the primary frequencies are required if a beat note is to be produced at the basilar 
membrane. Under these specific conditions only hearing of beat notes seems possible at all in Horseshoe Bats.

The high thresholds for low pure tone frequencies which restrict the hearing of beat notes to highly specified conditions, the mere existence of the Doppler effect compensation and the elaborate acoustical filter for echo frequencies at $83.3 \mathrm{kHz}$ make it unlikely that beat note frequencies are processed for echolocation.

\section{References}

Grinnell, A. D.: Comparative auditory neurophysiology of neotropical bats employing different echolocation signals. Z. vergl. Physiol. 68, 117-153 (1970). Kay, L.: Perception of distance in animal echolocation. Nature (Lond.) 190, $361-362$ (1961).

- A plausible theory of the bat's echolocation acuity. Animal Behav. 10, 34-41 (1962).

Neuweiler, G.: Neurophysiologische Untersuchungen zum Echoortungssystem der Großen Hufeisennase Rhinolophus ferrum equinum. Z. vergl. Physiol. 67, 273-306 (1970).

Pye, J. D.: A theory of echolocation by bats. J. Laryng. Otol. 74, 718-729 (1960).

- Perception of distance in animal echolocation. Nature (Lond.) 190, 362-363 (1961).

Schnitzler, H. U.: Die Ultraschall-Ortungslaute der Hufeisen-Fledermäuse in verschiedenen Orientierungssituationen. Z. vergl. Physiol. 57, 376-408 (1968).

- Echoortung bei der Fledermaus Chilonycteris rubiginosa. Z. vergl. Physiol. 68, 25-38 (1970).

Tonndorf, J.: Beats in cochlear models. J. acoust. Soc. Amer. 31, 608-619 (1959).

Dr. Gerhard Neuweiler

Zoophysiologisches Institut der Universität

BRD-7400 Tübingen, Köllestr. 23

Germany 